\title{
The Potential of Diffusion-Weighted Magnetic Resonance Imaging for Predicting the Outcomes of Chronic Subdural Hematomas
}

\author{
Seung-Hwan Lee, M.D., ${ }^{1}$ Jong-Il Choi, M.D, Ph.D., ${ }^{2}$ Dong-Jun Lim, M.D, Ph.D.,' Sung-Kon Ha, M.D, Ph.D., \\ Sang-Dae Kim, M.D, Ph.D., ${ }^{1}$ Se-Hoon Kim, M.D, Ph.D. ${ }^{1}$ \\ Department of Neurosurgery, Korea University Medical Center, Ansan, Korea \\ Department of Neurosurgery, ${ }^{2}$ Hallym University Kangnam Sacred Heart Hospital, Hallym University College of Medicine, Seoul, Korea
}

Objective : Diffusion-weighted magnetic resonance imaging (DW-MRI) has proven useful in the study of the natural history of ischemic stroke. However, the potential of DW-MRI for the evaluation of chronic subdural hematoma (CSDH) has not been established. In this study, we investigated DW-MRI findings of CSDH and evaluated the impact of the image findings on postoperative outcomes of CSDH.

Methods : We studied $131 \mathrm{CSDH}$ patients who had undergone single burr hole drainage surgery. The images of the subdural hematomas on preoperative DW-MRI and computed tomography (CT) were divided into three groups based on their signal intensity and density : 1) homogeneous (iso or low) density on $\mathrm{CT}$ and homogeneous low signal intensity on DW-MRl; 2) homogeneous (iso or low) density on CT and mixed signal intensity on DW-MRl; and 3) heterogeneous density on CT and mixed signal intensity on DW-MRI. On the basis of postoperative CT, we also divided the patients into 3 groups of surgical outcomes according to residual hematoma and mass effect.

Results : Analysis showed statistically significant differences in surgical ( $A$ to $B: p<0.001, A$ to $C: p<0.001, B$ to $C: p=0.129$ ) and functional ( $A$ to $B: p=0.039$, $A$ to $C: p<0.001, B$ to $C: p=0.108$ ) outcomes and treatment failure rates $(A$ to $B: p=0.037$, $A$ to $C: p=0.03$, $B$ to $C: p=1$ ) between the study groups. In particular, group $B$ and group $C$ showed worse outcomes and higher treatment failure rates than group $A$.

Conclusion : CSDH with homogeneous density on CT was characterized by signal intensity on DW-MRI. In CSDH patients, performing DW-MRI as well as CT helps to predict postoperative treatment failure or complications.

Key Words : Hematoma, Subdural, Chronic · Diffusion magnetic resonance imaging.

- Received : June 22, 2016 • Revised : December 1, 2016 •Accepted : March 3, 2017

- Address for reprints : Jong-II Choi, M.D, Ph.D.

Department of Neurosurgery, Hallym University Kangnam Sacred Heart Hospital, 1 Singil-ro, Yeongdeungpo-gu, Seoul 07441, Korea

Tel : +82-2-829-5179, Fax : +82-2-829-0219, E-mail : thlthd@korea.ac.kr

This is an Open Access article distributed under the terms of the Creative Commons Attribution Non-Commercial License (http://creativecommons.org/licenses/by-nc/4.0) which permits unrestricted non-commercial use, distribution, and reproduction in any medium, provided the original work is properly cited. 


\section{INTRODUCTION}

Chronic subdural hematoma (CSDH) is a common intracranial hemorrhagic disease and is relatively more prevalent in geriatric patients ${ }^{4,16)}$. Clinical presentations in CSDH patients generally include headache, dysarthria, and hemiparesis, which are similar to symptoms of cerebral infarction ${ }^{3,4)}$. Although there is occasionally no obvious history, $60-80 \%$ of cases of CSDH are due to head trauma ${ }^{4,17)}$. Therefore, in patients without a history of trauma, clinical symptoms alone may be difficult to differentiate between CSDH and cerebral ischemic stroke. Diffusion-weighted magnetic resonance imaging (DW-MRI) has proven useful in the study of the natural history of ischemic stroke and is currently a promising technique for the early detection of cerebral infarction in routine clinical practice ${ }^{3,12)}$. Consequently, many CSDH patients undergo DW-MRI as the first diagnostic imaging study because of the need to differentiate between CSDH and cerebral infarction, and several cases have been confirmed to be CSDH using DW-MRI.

Some institutions treat CSDH based on computed tomography (CT) findings, and there are several reports of treatment outcomes associated with features of hematoma on $\mathrm{CT}^{4,24)}$. However, there are few studies of treatment outcomes related to signal intensity of hematoma on DW-MRI. CT scan remains the most important imaging method in the initial evaluation of $\mathrm{CSDHs}^{15}$. It is a faster, cheaper and more available technique than DW-MRI. However, DW-MRI provides more precise localization and determination of the extent of hematoma and its mass effect on adjacent structures ${ }^{7}$. DW-MRI is more advantageous in the case of isodense and heterodence CSDHs on CT scans ${ }^{7,22)}$. CT and DW-MRI of CSDHs detect various patterns, which can be attributed to many factors including the age of the hematoma, the presence of rebleeding and the hematocrit status of the patient.

The purpose of this study is to predict the course and prognosis of treatment in patients with CSDH through DW-MRI. We reviewed preoperative DW-MRI, CT, and postoperative CT images in 131 patients with $\mathrm{CSDH}$ who underwent burr hole drainage surgery at a single medical center. We divided these patients into three groups based on the signal intensity on DW-MRI and features of hematoma on CT and investigated treatment outcomes in each group to determine the clinical significance of DW-MRI in CSDH patients.

\section{MATERIALS AND METHODS}

We studied 131 consecutive patients with CSDH at our institution from January 2012 to June 2015, and who had received single burr-hole drainage surgery. CSDH was defined as the radiographic finding of a subdural fluid collection surrounded by a typical neomembrane that contained dark red or brown liquid blood ${ }^{4,6,9,11,12)}$. Diagnosis was confirmed by preoperative CT and DW-MRI in all patients. We performed DW-MRI after a CT scan for those patients with proven history of head trauma within 3 months and who showed neurologic deficits such as headache, dysarthria and hemiparesis. For those without any trauma history, we performed DWMRI first, then followed by CT scan on the day of, or 1 day prior to surgery.

Preoperative DW-MRI was performed on admission using a 3.0-T superconducting system (3T Achieva X-series; Philips Healthcare, Best, The Netherlands). Single-shot echo-planar DW-MRI was obtained in three orthogonal motion probing gradients (repetition time/echo time $=9800 / 76 \mathrm{~ms}$, field of view $220 \times 220 \mathrm{~mm}$, slice thickness $3 \mathrm{~mm}$ with a $1 \mathrm{~mm}$ gap, matrix $174 \times 174 \mathrm{~mm}, \mathrm{MPG}$ of $1 \mathrm{mT} / \mathrm{m}$, and maximum b-factor of $1000 \mathrm{~s} / \mathrm{cm}^{2}$ ). Postoperative CT was obtained at 1,4 , and 8 weeks, and 6 months after surgery.

All patients underwent a single burr hole procedure under general anesthesia. A burr hole trephination was placed over
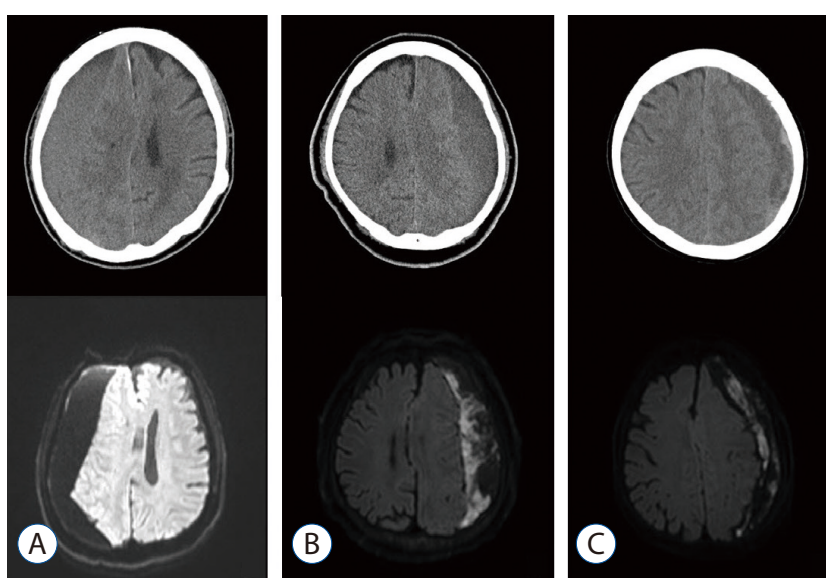

Fig. 1. Appearance of subdural hematomas on diffusion-weighted magnetic resonance imaging (DW-MRI) and preoperative computed tomography (CT). A : Homogeneous density on CT and homogeneous signal intensity on DW-MRI. B : Homogeneous density on CT and mixed signal intensity on DW-MRI. C : Heterogeneous density on CT and mixed signal intensity on DW-MRI. 
the maximum thickness of the hematoma, usually at the parietal eminence of the parietal bone. Dura and outer pseudomembranes were opened in a cruciate fashion. The hematoma was removed by rinsing with warm saline and gentle aspiration, and the cavity was irrigated and filled with sterile saline. A closed drainage system with a silicone catheter was left in the subdural space for 2 or 3 days ${ }^{18,20)}$.

The radiologic characteristics of the subdural hematomas on preoperative CT and DW-MRI were divided into three groups by two neurosurgeons in our institution, based on their density and signal intensity : 1) homogeneous (iso or low) density on CT and homogeneous low signal intensity on DWMRI; 2) homogeneous (iso or low) density on CT and mixed signal intensity on DW-MRI; and 3) heterogeneous density on CT and mixed signal intensity on DW-MRI (Fig. 1). Two neurosurgeons assessed imaging findings of CT and DW-MRI independently without being influenced by each other. If the image classification of the two neurosurgeons did not match, we reviewed and discussed the image together to derive the agreed classification.

The results of surgical outcomes and functional prognosis were evaluated and compared using methods from previous studies ${ }^{1,6,14,19)}$. The pre- and postoperative (follow-up period of 6 months) hematoma thickness was measured using CT to calculate the brain expansion rate as : (preoperative hematoma thickness-postoperative hematoma thickness)/preoperative hematoma thickness $\times 100$ (\%). Surgical outcome was defined by the degree of brain expansion as good $(>80 \%)$, partial (50$80 \%$ ), or none $(<50 \%)^{14,19)}$. Good functional prognosis was defined using a modified Rankin Scale (mRS) of $0-2$, while poor functional prognosis was graded as $3-6$, after 3 months ${ }^{14,19)}$.
Treatment failure was defined as the occurrence of symptoms and signs attributable to an ipsilateral hematoma seen on CT within the follow-up period of 6 months ${ }^{6,14,19)}$.

Statistical analyses were performed using the Statistical Package for the Social Sciences version 18.0 for Windows (SPSS Inc., Chicago, IL, USA). Chi-squared test and Fisher's exact test were used to assess the postoperative outcomes and treatment failure, and to compare the groups in this study. Differences were considered significant at $p$ values of less than or equal to 0.05 .

\section{RESULTS}

\section{Baseline characteristics of CSDH patients}

A total of 131 patients were enrolled in this study, with 58, 30 , and 43 patients in groups $\mathrm{A}, \mathrm{B}$, and $\mathrm{C}$, respectively. The mean age of all patients was $68 \pm 17$ years and the mean age in groups A, B, and C was $70 \pm 14$ years, $64 \pm 20$ years, and $68 \pm 16$ years. Of 131 cases, 85 were male (64.9\%) and 46 were female (35.1\%), with a patient sex distribution as follows : group A, 37 males and 15 females; group B, 22 males and 13 females; and group C, 26 males and 18 females. Fifty-one of the patients (38.9\%) had a history of hypertension, 35 (26.7\%) had diabetes mellitus, and $35(26.7 \%)$ were on antiplatelet or anticoagulant drugs; $41.4 \%, 26.7 \%$, and $44.2 \%$ of patients in groups A, B, and $\mathrm{C}$ had been treated for hypertension, and 22.4\%, 33.3\%, and $27.9 \%$ for diabetes mellitus, respectively. Antiplatelet or anticoagulant drugs were taken by $22.4 \%, 30.0 \%$, and $30.2 \%$ of patients in groups $\mathrm{A}, \mathrm{B}$, and $\mathrm{C}$, respectively. There was a definite history of head trauma in 71 cases (54.2\%); 51.7\%,

Table 1. Baseline characteristics of groups in this study

\begin{tabular}{|c|c|c|c|c|c|}
\hline Factor & Overall patients & Group A & Group B & Group C & $p$-value \\
\hline No. of patients & 131 & 58 & 30 & 43 & \\
\hline Mean age (years) & 68 & 70 & 64 & 68 & 0.488 \\
\hline Sex & & & & & 0.431 \\
\hline Male & 85 & 37 & 22 & 26 & \\
\hline Female & 46 & 15 & 13 & 18 & \\
\hline Hypertension & 51 & 24 & 8 & 19 & 0.863 \\
\hline Diabetes mellitus & 35 & 13 & 10 & 12 & 0.497 \\
\hline History of trauma & 71 & 30 & 20 & 21 & 0.861 \\
\hline Antiplatelet or anticoagulant drug & 35 & 13 & 9 & 13 & 0.365 \\
\hline
\end{tabular}



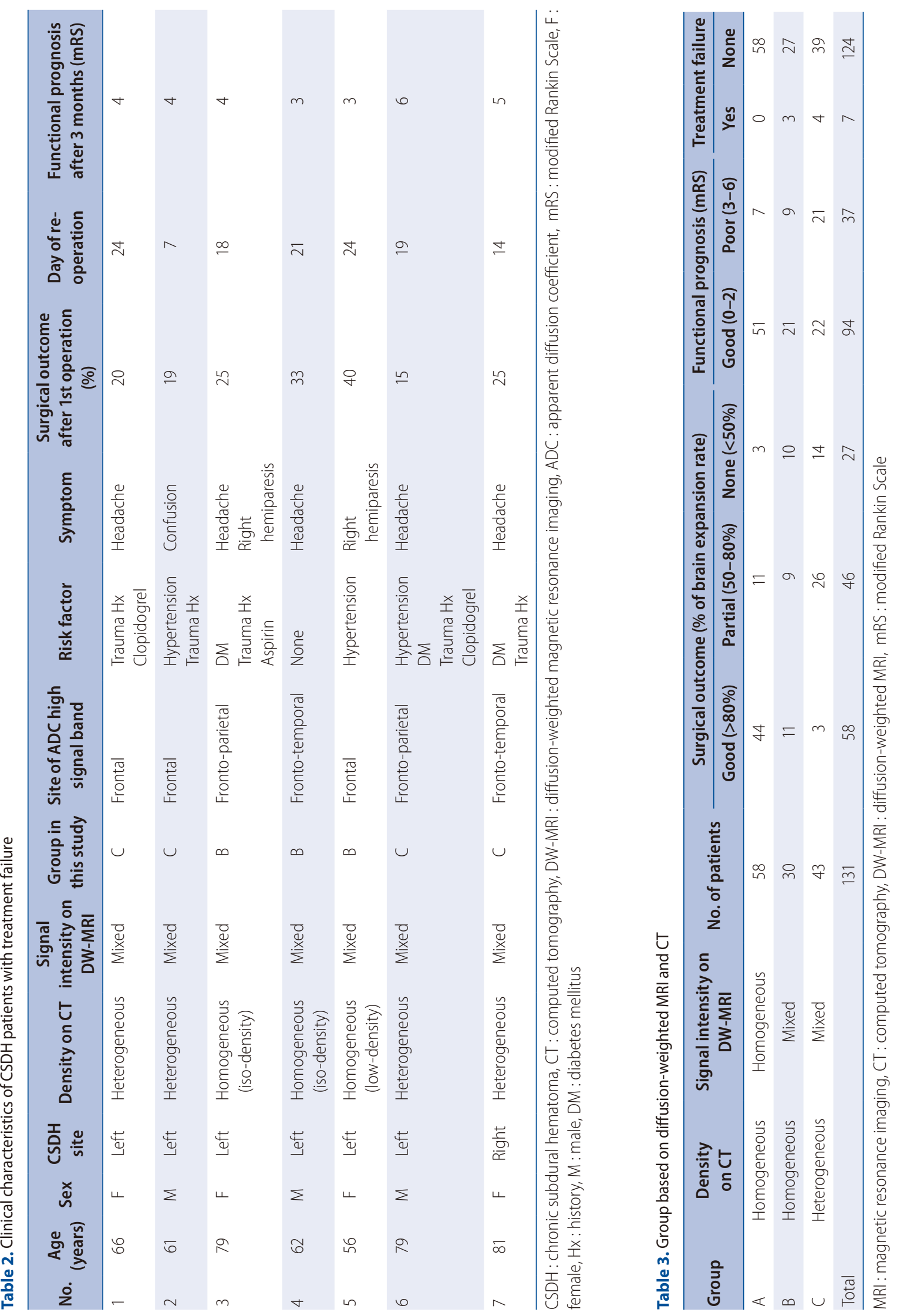
Table 4. Statistical comparisons between groups

\begin{tabular}{lccc}
\hline Group & \multicolumn{3}{c}{$p$-value } \\
\cline { 2 - 4 } & $\begin{array}{c}\text { Surgical } \\
\text { outcome }\end{array}$ & $\begin{array}{c}\text { Functional } \\
\text { prognosis }\end{array}$ & Treatment failure \\
\hline A to B & $<0.001$ & 0.037 \\
A to C & $<0.001$ & $<0.001$ & 0.03 \\
B to C & 0.129 & 0.108 & 1.000 \\
\hline
\end{tabular}
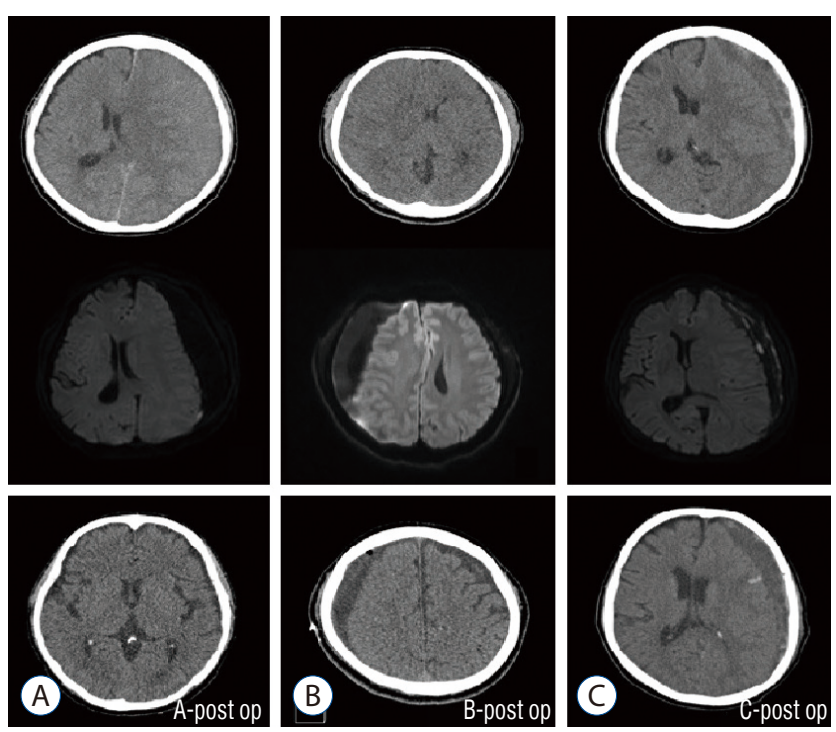

Fig. 2. Postoperative computed tomography of each group in this study. A : Favorable surgical outcomes. B and C : Poor surgical outcomes.

$66.7 \%$, and $48.8 \%$ of patients in groups A, B, and C had a history of trauma.

No statistical difference between groups was noted regarding baseline characteristics such as age, sex, and history of hypertension, diabetes mellitus, trauma, or use of antiplatelet or anticoagulant drugs (Table 1).

\section{Treatment failure}

The rate of treatment failure was $8.4 \%$ (7 of 131) after the first burr hole operation for CSDH (Table 2). Treatment failures in groups $\mathrm{A}, \mathrm{B}$, and $\mathrm{C}$ were reported as $0(0.0 \%), 3$ $(10.0 \%)$, and $4(9.3 \%)$, respectively. Statistical evaluation of treatment failure rates showed a significant difference between groups A and B and groups A and C $(p=0.037$ and $p=0.03$, respectively), but there was no difference between groups $\mathrm{B}$ and $\mathrm{C}(p=1)$ (Tables 3 and 4$)$.

\section{Analysis of surgical outcome for brain expansion}

Analysis of surgical outcomes was performed in 131 patients; 58 patients (44.2\%) had a good outcome, $46(35.1 \%)$ had a partial outcome, and 27 (20.6\%) had none. In group A, 44 patients (75.9\%) had a good outcome, 11 (19.0\%) had a partial outcome, and 3 (5.2\%) had none. In group B, 11 (36.7\%) had a good outcome, $9(30.0 \%)$ had a partial outcome, and 10 (33.3\%) had none. In group C, 3 (7.0\%) had a good outcome, $26(60.4 \%)$ had a partial outcome, and 14 (32.6\%) had none. Statistical comparison of surgical outcomes showed a significant difference between groups $\mathrm{A}$ and $\mathrm{B}$ and groups $\mathrm{A}$ and $\mathrm{C}$ ( $p<0.001$ for both comparisons). However, there was no statistically significant difference between groups $\mathrm{B}$ and $\mathrm{C}$ $(p=0.129)$. Thus, groups B and C had poorer surgical outcomes than group A (Tables 3 and 4). Fig. 2 shows the postoperative CT results for each group.

\section{Functional outcome}

Overall, 37 patients $(28.2 \%)$ had poor functional outcomes. In group A, 51 patients $(88.0 \%)$ had a good functional outcome and $7(12.1 \%)$ had a poor outcome. In group B, 21 patients $(70.0 \%)$ had a good outcome and $9(30.0 \%)$ had a poor outcome. In group C, 22 patients (51.2\%) had a good outcome and $21(48.8 \%)$ had a poor outcome. Statistical comparison of functional outcomes showed a significant difference between groups A and B and groups A and C ( $p=0.039$ and $p<0.001$, respectively), but there was no significant difference between groups B and C ( $p=0.108)$. Therefore, groups B and C had poorer functional outcomes than group $\mathrm{A}$, similar to the results for surgical outcome and treatment failure rates (Tables 3 and 4).

\section{DISCUSSION}

\section{CT for CSDH}

CT is useful for predicting CSDH outcome in several ways. CT is considered the most important diagnostic tool for $\mathrm{CSDH}$, although conventional MRI has superior image quality ${ }^{4,6,16)}$. In previous studies, it was reported that hematomas with homogeneous density are less likely to recur and hematomas with heterogeneous density have a greater likelihood of treatment failure $e^{1,4,9,13,19,24)}$. Moreover, it has been reported that 
Table 5. Outcome based on CT or DW-MRI only

\begin{tabular}{|c|c|c|c|c|c|c|c|c|c|c|c|}
\hline & \multirow{2}{*}{$\begin{array}{l}\text { No. of } \\
\text { patients }\end{array}$} & \multicolumn{4}{|c|}{$\begin{array}{c}\text { Surgical outcome } \\
\text { (\% of brain expansion rate) }\end{array}$} & \multicolumn{3}{|c|}{ Functional prognosis (mRS) } & \multicolumn{3}{|c|}{ Treatment failure } \\
\hline & & $\begin{array}{l}\text { Good } \\
(>80 \%)\end{array}$ & $\begin{array}{c}\text { Partial } \\
(50-80 \%)\end{array}$ & $\begin{array}{c}\text { None } \\
(<50 \%)\end{array}$ & $p$-value & $\begin{array}{l}\text { Good } \\
(0-2)\end{array}$ & Poor (3-6) & $p$-value & Yes & None & $p$-value \\
\hline \multicolumn{12}{|l|}{ Density on CT } \\
\hline Homogeneous & 88 & 55 & 20 & 13 & 0.506 & 72 & 16 & $<0.001$ & 3 & 85 & 0.095 \\
\hline Heterogeneous & 43 & 3 & 26 & 14 & & 22 & 21 & & 4 & 39 & \\
\hline Total & 131 & 58 & 46 & 27 & & 94 & 37 & & 7 & 124 & \\
\hline \multicolumn{12}{|l|}{$\begin{array}{l}\text { Signal intensity } \\
\text { on DW-MRI }\end{array}$} \\
\hline Homogeneous & 58 & 44 & 11 & 3 & $<0.001$ & 51 & 7 & 0.002 & 0 & 58 & 0.017 \\
\hline Mixed & 73 & 14 & 35 & 24 & & 43 & 30 & & 7 & 66 & \\
\hline Total & 131 & 58 & 46 & 27 & & 94 & 37 & & 7 & 124 & \\
\hline
\end{tabular}

CT : computed tomography, DW-MRI : diffusion-weighted magnetic resonance imaging, mRS : modified Rankin Scale

layering and mixed-density hematoma types have a marked tendency to rebleed, and that mixed- and high-density hematomas showed recent bleeding ${ }^{9,13,19,24)}$. The incidence of $\mathrm{CSDH}$ recurrence in the high- and mixed-density groups was significantly higher than that in the low- and iso-density groups $^{4,9,13,19,24)}$. However, it was not possible to precisely predict treatment failure or postoperative outcomes based on preoperative CT findings alone, because the classification of CT findings can be subjective.

In our study, the treatment failure rate in the heterogeneous density group (9.3\%) based on preoperative CT scans was higher than that in the homogenous density group (3.4\%). However, other than for functional outcome, there was no statistically significant difference between the two groups. The prediction of outcomes and treatment failure rates based on preoperative CT did not reflect the true treatment failure rates and postoperative surgical and functional outcomes in CSDH (Table 5).

\section{DW-MRI for CSDH}

DW-MRI is based on the movement of water molecules, and some changes in DW-MRI are indicative of changes in the water molecules ${ }^{3,10)}$. Structured hematomas usually involve thick, fibrous, collagenous membranes and blood clots. Calcium, hemosiderin, and cholesterol accumulate in the membranes and trabeculae. In contrast, the liquid component appears as low signal intensity on DW-MRI, similar to that of normal cerebrospinal fluid $^{5,10,12)}$. Further subdivided by apparent diffusion coefficient (ADC) value, DW-MRI shows the liquid component of a subdural hematoma as low intensity, with a higher ADC value than the solid component, and the brain tissue as high intensity, ${ }^{5,10,11)}$. The ADC values of low-intensity areas on DW-MRI reflect a combination of cerebrospinal fluid and extracellular methemoglobin from the blood clot $^{5,11)}$. The high-intensity areas in a subacute hematoma on DW-MRI are believed to mainly consist of extracellular methemoglobin ${ }^{11)}$. The low-intensity areas also match the high-intensity areas on conventional MRI (T1- and T2-weighted imaging $)^{6,10,23)}$. The low-intensity areas, based on ADC values, are considered to represent fluid containing extracellular methemoglobin, and are influenced by molecular water diffusion. A subdural hyperintense band on DW-MRI is considered to reflect comparatively recent hemorrhage from the outer membrane, and the ADC value of the hyperintense band is close to that reported for intracellular and/or extracellular methemoglobin $^{5,10,11)}$. The subdural hyperintense band on DW-MRI is conspicuous, in contrast to adjacent normal tissues, because the liquid components in CSDH appear with low intensity, and the dura mater appears with iso- and/or low intensity ${ }^{5,10,11)}$. Consequently, a mixed signal intensity area and hyperintense band on DW-MRI indicate that CSDH has a solid component or relatively fresh bleeding.

In our study, the postoperative outcomes and treatment failure rates of cases with mixed signal intensity were significantly different from those of the homogeneous type (Table 5). 
Several studies have shown that the type of signal intensity in DW-MRI is correlated with the risk of rebleeding ${ }^{3,5,10,11,12,23)}$.

Moreover, in some cases, CSDH with homogeneous density on CT shows mixed signal intensity on DW-MRI. Treatment failure rates in these cases are similar to those in cases of heterogeneous density on CT and mixed signal intensity on DWMRI (Table 3). This may indicate that burr hole surgery for CSDH requires preoperative DW-MRI to predict the risk of treatment failure and postoperative outcomes.

The benefits of conventional MRI versus CT in the evaluation of CSDH have already been outlined, especially in cases with homogeneous density on $\mathrm{CT}^{23)}$. However, the longer time to acquire results and the higher cost are some of the advantages of conventional MRI ${ }^{6,12,23)}$. DW-MRI and measurement of ADC values in CSDH were useful for rapid identification of solid and liquid hematomas in the subdural space ${ }^{10-12)}$.

\section{Other risk factors}

Previous studies did not show a difference in treatment failure rates between male patients and female patients with $\mathrm{CSDH}^{4,8,16,24)}$, but older patients had a higher risk of treatment failure ${ }^{4,9,14,16,24)}$. There are few studies in the literature regarding the risk of high treatment failure rates in CSDH patients with hypertension ${ }^{4,14,1724)}$. Diabetic patients have capillary vasculopathy, and there is a significant capillary network on the outer membrane in $\mathrm{CSDH}^{4,9,17,24)}$. Consequently, the vasculopathy may lead to hematoma recurrence.

Other studies have reported that trauma is the most important factor in $\mathrm{CSDH}$, with a greater likelihood of recurrence in patients with a history of trauma ${ }^{4,9,14,24)}$. Moreover, the use of antiplatelet and anticoagulant drugs is strongly related to the occurrence and recurrence of $\mathrm{CSDH}^{2,4,17,21)}$.

There are several limitations of the current study. First, the number of patients was small. Second, this was a retrospective study and is therefore subject to potential sources of selection bias, in addition, there was no control group.

\section{CONCLUSION}

In this study, we divided patients into three groups, based on DW-MRI and CT findings, with the following results. CSDH with homogeneous density on CT showed mixed signal intensity on DW-MRI in some cases. In mixed signal intensity types of DW-MRI, treatment failure and poor outcome rates in CSDH were increased. Thus, in CSDH patients, performing DW-MRI helped to detect cerebral infarction at an early stage and predict postoperative treatment failure or outcome. Compared with conventional MRI, DW-MRI also had advantages with regard to the cost and time required to obtain results.

\section{PATIENT CONSENT}

The patient provided written informed consent for the publication and the use of their images.

\section{References}

1. Amirjamshidi A, Abouzari M, Eftekhar B, Rashidi A, Rezaii J, Esfandiari K, et al. : Outcomes and recurrence rates in chronic subdural haematoma. Br J Neurosurg $21: 272-275,2007$

2. Aspegren OP, Åstrand R, Lundgren MI, Romner B : Anticoagulation therapy a risk factor for the development of chronic subdural hematoma. Clin Neurol Neurosurg 115 : 981-984, 2013

3. Augustin M, Bammer R, Simbrunner J, Stollberger R, Hartung HP, Fazekas $F$ : Diffusion-weighted imaging of patients with subacute cerebral ischemia: comparison with conventional and contrast-enhanced MR imaging. AJNR Am J Neuroradiol 21 : 1596-1602, 2000

4. Borger V, Vatter H, Oszvald Á, Marquardt G, Seifert V, Güresir E : Chronic subdural haematoma in elderly patients: a retrospective analysis of 322 patients between the ages of 65-94 years. Acta Neurochir (Wien) 154 : 1549-1554, 2012

5. Fainardi E, Borrelli M, Saletti A, Sarubbo S, Roversi G, Bernardoni A, et al. : Temporal changes in perihematomal apparent diffusion coefficient values during the transition from acute to subacute phases in patients with spontaneous intracerebral hemorrhage. Neuroradiology 55 : 145-156, 2013

6. Goto H, Ishikawa O, Nomura M, Tanaka K, Nomura S, Maeda K : Magnetic resonance imaging findings predict the recurrence of chronic subdural hematoma. Neurol Med Chir (Tokyo) 55 : 173-178, 2015

7. Hosoda K, Tamaki N, Masumura M, Matsumoto S, Maeda F : Magnetic resonance images of chronic subdural hematomas. J Neurosurg 67 : 677-683, 1987

8. Jamjoom A, Nelson R, Stranjalis G, Wood S, Chissell H, Kane N, et al. : Outcome following surgical evacuation of traumatic intracranial haematomas in the elderly. Br J Neurosurg 6 : 27-32, 1992

9. Ko BS, Lee JK, Seo BR, Moon SJ, Kim JH, Kim SH : Clinical analysis of risk factors related to recurrent chronic subdural hematoma. J Korean Neurosurg Soc 43 : 11-15, 2008

10. Kuwahara S, Fukuoka M, Koan Y, Miyake H, Ono Y, Moriki A, et al. : Subdural hyperintense band on diffusion-weighted imaging of chronic 
subdural hematoma indicates bleeding from the outer membrane. Neurol Med Chir (Tokyo) 45 : 125-131, 2005

11. Kuwahara S, Fukuoka M, Koan Y, Miyake H, Ono Y, Moriki A, et al. : Diffusion-weighted imaging of traumatic subdural hematoma in the subacute stage. Neurol Med Chir (Tokyo) 45 : 464-469, 2005

12. Kuwahara S, Miyake H, Fukuoka M, Koan Y, Ono Y, Moriki A, et al. : Diffusion-weighted magnetic resonance imaging of organized subdural hematoma--case report. Neurol Med Chir (Tokyo) 44 : 376-379, 2004

13. Kwon TH, Park YK, Lim DJ, Cho TH, Chung YG, Chung HS, et al. : Chronic subdural hematoma: evaluation of the clinical significance of postoperative drainage volume. J Neurosurg 93 : 796-799, 2000

14. Leroy HA, Aboukaïs R, Reyns $N$, Bourgeois $P$, Labreuche J, Duhamel A, et al. : Predictors of functional outcomes and recurrence of chronic subdural hematomas. J Clin Neurosci 22 : 1895-1900, 2015

15. Markwalder TM. Chronic subdural hematomas: a review. J Neurosurg 54 : 637-645, 1981

16. Nakaguchi $\mathrm{H}$, Tanishima T, Yoshimasu N : Factors in the natural history of chronic subdural hematomas that influence their postoperative recurrence. J Neurosurg 95 : 256-262, 2001

17. Ohba S, Kinoshita Y, Nakagawa T, Murakami $H$ : The risk factors for recurrence of chronic subdural hematoma. Neurosurg Rev 36 : 145-149; discussion 149-150, 2013

18. Richter HP, Klein HJ, Schäfer M : Chronic subdural haematomas treated by enlarged burr-hole craniotomy and closed system drainage. Retrospective study of 120 patients. Acta Neurochir (Wien) 71 : 179-188, 1984

19. Ro HW, Park SK, Jang DK, Yoon WS, Jang KS, Han YM : Preoperative predictive factors for surgical and functional outcomes in chronic subdural hematoma. Acta Neurochir (Wien) 158 : 135-139, 2016

20. Santarius T, Kirkpatrick PJ, Ganesan D, Chia HL, Jalloh I, Smielewski P, et al. : Use of drains versus no drains after burr-hole evacuation of chronic subdural haematoma: a randomised controlled trial. Lancet 374 : 1067-1073, 2009

21. Santarius T, Kirkpatrick PJ, Kolias AG, Hutchinson PJ : Working toward rational and evidence-based treatment of chronic subdural hematoma. Clin Neurosurg 57 : 112-122, 2010

22. Sipponen JT, Sepponen RE, Sivula A. Chronic subdural hematoma: demonstration by magnetic resonance. Radiology $150:$ 79-85, 1984

23. Tsutsumi K, Maeda K, lijima A, Usui M, Okada Y, Kirino T : The relationship of preoperative magnetic resonance imaging findings and closed system drainage in the recurrence of chronic subdural hematoma. J Neurosurg $87: 870-875,1997$

24. Tugcu B, Tanriverdi O, Baydin S, Hergunsel B, Günaldi Ö, Ofluoglu E, et al. : Can recurrence of chronic subdural hematoma be predicted? A retrospective analysis of 292 cases. J Neurol Surg A Cent Eur Neurosurg $75: 37-41,2014$ 